\title{
Avaliação da atividade larvicida de extratos obtidos do caule de Croton linearifolius Mull. Arg. (Euphorbiaceae) sobre larvas de Aedes aegypti (Linnaeus, 1762) (Diptera: Culicidae)
}

\author{
Sandra Lúcia da Cunha e Silva * \\ Simone Andrade Gualberto \\ Karine da Silva Carvalho \\ Daniela Deitos Fries \\ Universidade Estadual do Sudoeste da Bahia, Campus de Itapetinga \\ Praça Primavera, 40, Bairro Primavera, CEP 45700-000, Itapetinga - BA, Brasil \\ * Autor para correspondência \\ cunhasl@pq.cnpq.br
}

Submetido em 24/07/2013

Aceito para publicação em 12/03/2014

\section{Resumo}

Dentre os problemas de saúde pública que acometem a população humana, em nível nacional e mundial, destaca-se a dengue, cujo principal vetor do vírus é o Aedes aegypti (Linnaeus, 1762). Para a redução da densidade populacional desse vetor, recomenda-se o controle integrado e, como parte desse controle, o uso de inseticidas. No entanto, tal prática tem levado à seleção de populações de insetos resistentes. Dessa forma, faz-se necessário buscar inseticidas alternativos que possam contribuir no controle desse vetor. O objetivo desse estudo foi avaliar o potencial larvicida do extrato obtido do caule de Croton linearifolius contra larvas de A. aegypti, bem como realizar uma prospecção fitoquímica do extrato etanólico. O extrato etanólico e as frações hexânica, diclorometânica e hidroalcóolica, além do acetato de etila, foram testadas sobre larvas de terceiro instar de $A$. aegypti. Os ensaios biológicos revelaram a efetividade larvicida de extrato do caule de $C$. linearifolius contra o A. aegypti, especialmente as frações diclorometânica e hexânica. A prospecção fitoquímica demonstrou a presença no extrato etanólico de ácidos fixos fortes, alcaloides, catequinas, esteroides livres, flavonois, flavonas, resinas, taninos condensados e xantonas.

Palavras-chave: Caatinga; Controle; Inseticida botânico

\section{Abstract}

Evaluation of the larvicidal activity of extracts obtained from the stem of Croton linearifolius Mull. Arg. (Euphorbiaceae) against larvae of Aedes aegypti (Linnaeus, 1762) (Diptera: Culicidae). Among the public health problems affecting the human population, at the national and world levels, dengue fever stands out, whose primary vector of the virus is Aedes aegypti (Linnaeus, 1762). For reducing the population density of this vector, an integrated control is recommended and, as an element of this control, the use of insecticides. However, this practice has led to the selection of resistant insect populations. Thus, there is a need to seek alternative insecticides that can contribute to control this vector. This study aimed to evaluate the larvicidal potential of an extract obtained from the stem of Croton linearifolius against larvae of A. aegypti, 
as well as conduct a phytochemical screening of the ethanolic extract. The ethanolic extract and the hexane, dichloromethanic, and hydroalcoholic fractions, besides ethyl acetate, were tested on third-instar larvae of $A$. aegypti. The biological assays revealed the larvicidal effectiveness of the extract of the stem of $C$. linearifolius against $A$. aegypti, especially the dichloromethanic and hexane fractions. Phytochemical screening showed the presence in the ethanolic extract of strong fixed acids, alkaloids, catechins, free steroids, flavonoids, flavones, resins, condensed tannins, and xanthones.

Key words: Botanical insecticide; Caatinga; Control

\section{Introdução}

Segundo a Organização Mundial da Saúde (OMS), a dengue é um dos principais problemas de saúde pública no mundo e estima-se que 50 milhões de pessoas sejam infectadas anualmente, com cerca de 550.000 hospitalizações e 20.000 óbitos. A transmissão ocorre pela picada da fêmea do mosquito Aedes aegypti (Linnaeus, 1762), contaminado com o vírus do gênero Flavivírus. Um agravante deste quadro é a ausência de vacinas para a sua prevenção (WHO, 2008).

O principal objetivo da maioria dos programas de controle é reduzir a densidade populacional do vetor do dengue, utilizando métodos que incluem a eliminação ou controle de criadouros (OPS/OMS, 2010), o controle químico das larvas (WHO, 2009), o uso de agentes biológicos, bem como a aplicação de adulticidas (OPS/ OMS, 2010). Em 2012, a OMS lançou a estratégia global para a prevenção e controle da dengue. Dentre essas ações, que deverão ser sustentáveis, economicamente viáveis e ao mesmo tempo ecologicamente corretas, destacase o controle integrado, cujo princípio orientador é a prevenção e a vigilância entomológica e epidemiológica (WHO, 2012).

No que diz respeito à vigilância entomológica o uso contínuo de inseticidas tem levado a seleção de populações de insetos resistentes. Em muitos países foram detectadas populações de $A$. aegypti com níveis de resistência significativos aos organosfosforados, piretroides, carbamatos e organoclorados (OPS/OMS, 2010). Porphiro et al. (2011), analisando o status de susceptibilidade ao temephos em populações de $A$. aegypti, no estado do Paraná, verificaram um baixo nível de suscetibilidade a esse inseticida, nas populações avaliadas, contudo, os autores ressaltam a necessidade de um monitoramento contínuo dessas populações. Horta et al. (2011), em um estudo realizado com o objetivo de verificar a suscetibilidade do $A$. aegypti ao temephos, no município de Coronel Fabriciano, em Minas Gerais, destacam a necessidade, em virtude do processo de seleção já ter se iniciado nessa população, de se buscar novas estratégias para o controle desse vetor. Dessa forma, a busca por métodos alternativos para serem usados no controle do $A$. aegypti, que sejam eficientes, economicamente viáveis, biodegradáveis e mais seletivos torna-se essencial. Dentre essas alternativas destacam-se os inseticidas botânicos e nesse contexto o Brasil tem um grande potencial devido a sua rica biodiversidade florística.

O objetivo desse estudo foi avaliar o potencial larvicida de extratos obtidos do caule de Croton linearifolius, Mull. Arg., espécie encontrada na Floresta Nacional Contendas do Sincorá, situada no bioma caatinga (BRASIL, 2006), sobre larvas de A. aegypti, bem como realizar a prospecção fitoquímica do extrato etanólico.

\section{Material e Métodos}

As etapas da coleta e identificação de Croton linearifolius, bem como o depósito da exsicata no herbário da Universidade Estadual de Feira de Santana, sob o registro HUEFS 146620, foram supervisionadas por um botânico especialista. A coleta foi realizada no mês de outubro, no período matutino, na unidade de conservação Floresta Nacional Contendas do Sincorá, no Estado da Bahia (13 $58^{\prime} 10,8^{\prime \prime}$; $\left.41^{\circ} 07^{\prime} 7,6^{\prime \prime} \mathrm{W}\right)$.

Posteriormente, o caule foi pesado e cortado em pequenos fragmentos, os quais foram levados para uma estufa de circulação de ar, onde permaneceu durante 48 h, sob a temperatura de $50^{\circ} \mathrm{C}$. Em seguida, procedeuse a moagem do material vegetal através de moinhos 
de facas. O extrato etanólico foi obtido através do processo de percolação, utilizando-se etanol a 95\% e, em seguida, concentrado em evaporador rotatório. Após a realização da avaliação inseticida desse extrato, com a comprovação de seu potencial larvicida, realizou-se o fracionamento do mesmo, através da técnica líquidolíquido, utilizando-se, sucessivamente, os solventes hexano, diclorometano e acetato de etila, obtendo-se as frações hexânica, diclorometânica, acetato de etila e hidroalcóolica, as quais foram avaliadas posteriormente.

As larvas utilizadas nos bioensaios foram oriundas de uma colônia estabelecida no Laboratório de Pesquisa de Inseticidas Naturais, da Universidade Estadual do Sudoeste da Bahia, a partir de ovos da linhagem Rockefeller, cedidos pelo Laboratório de Fisiologia e Controle de Artrópodes Vetores (LAFICAVE), da Fundação Oswaldo Cruz, do Rio de Janeiro.

Para a realização dos bioensaios, utilizou-se larvas de terceiro instar de $A$. aegypti. Foram utilizadas nos ensaios biológicos cinco concentrações $(13,3 \mathrm{mg} / \mathrm{mL}$, $6,7 \mathrm{mg} / \mathrm{mL}, 4,0 \mathrm{mg} / \mathrm{mL}, 2,0 \mathrm{mg} / \mathrm{mL}$ e $0,7 \mathrm{mg} / \mathrm{mL}$ ), obtidas a partir de uma solução estoque de $400 \mathrm{mg} / \mathrm{mL}$. Cada tratamento foi composto por quatro repetições, tendo sido utilizado 30 larvas por repetição, totalizando 120 larvas por tratamento.

Como solvente para a solubilização do extrato etanólico, bem como para o controle, foi usado o etanol e água destilada, na proporção de 6:4. Para a solubilização das frações de acetato de etila, diclorometano, hexano e hidroalcoólica, e dos seus respectivos grupos controle, utilizou-se dimetilsulfóxido e água destilada, nas proporções de 4:6, 4:6, 7:3 e 6:4, respectivamente.

$\mathrm{O}$ experimento foi conduzido em uma sala climatizada, com temperatura de $27^{\circ} \mathrm{C}$ e umidade média de $70 \%$. As observações da mortalidade das larvas foram realizadas nos intervalos de 4, 8, 16 e 24 h, após o início do experimento.

Para o cálculo da concentração letal $50\left(\mathrm{CL}_{50}\right)$ foi usado o programa computacional POLO-PC (Probit for Logit Analysis). Os percentuais de mortalidade das larvas foram submetidos ao teste de Tukey, em nível de $5 \%$ de probabilidade.

Paralelamente aos ensaios biológicos foi realizada a prospecção fitoquímica do extrato etanólico do caule de C.linearifolius. A técnica de prospecção seguiu a metodologia preconizada por Matos (1988), que consistiu na preparação de um extrato hidrofílico (etanol/ água) e outro lipofílico (clorofórmio). Posteriormente, esses extratos foram submetidos a uma marcha analítica prospectiva com o objetivo de detectar os seguintes constituintes químicos: ácidos fixos fortes, alcaloide, antocianidina, antocianina, base quaternária, catequina, cumarina, esteroide livre, fenois, flavonois, flavanonois, flavanona, flavona, heterosídeo cianogênico, leucoantocianida, quinona, resina, saponina, tanino condensado, triterpenoide e xantona.

\section{Resultados}

O percentual de mortalidade larval ocasionado pelo extrato etanólico do caule de Croton linearifolius atingiu 50\% de mortalidade com $24 \mathrm{~h}$ de exposição, sendo a concentração de $13,3 \mathrm{mg} / \mathrm{mL}$ significativamente mais efetiva $(65,00 \%)$ quando comparada às demais concentrações e o grupo controle (Tabela 1).

TABELA 1: Percentual de mortalidade de larvas de terceiro instar de Aedes aegypti, em relação ao tempo de exposição às diferentes concentrações $(\mathrm{mg} / \mathrm{mL})$, do extrato etanólico do caule de Croton linearifolius.

\begin{tabular}{ccccc}
\hline $\begin{array}{c}\text { Concentrações } \\
(\mathbf{m g} / \mathbf{m L})\end{array}$ & \multicolumn{4}{c}{ Mortalidade (\%) } \\
\hline & $4 \mathrm{~h}$ & $8 \mathrm{~h}$ & $16 \mathrm{~h}$ & $24 \mathrm{~h}$ \\
\cline { 2 - 5 } 13,3 & $11,67 \mathrm{a}$ & $24,17 \mathrm{a}$ & $43,33 \mathrm{a}$ & $65,00 \mathrm{a}$ \\
6,7 & $3,33 \mathrm{~b}$ & $6,66 \mathrm{~b}$ & $10,83 \mathrm{~b}$ & $27,50 \mathrm{~b}$ \\
4,0 & $1,66 \mathrm{~b}$ & $0,83 \mathrm{~b}$ & $5,00 \mathrm{~b}$ & $12,50 \mathrm{bc}$ \\
2,0 & $0,83 \mathrm{~b}$ & $0,00 \mathrm{~b}$ & $3,33 \mathrm{~b}$ & $9,16 \mathrm{bc}$ \\
0,7 & $0,83 \mathrm{~b}$ & $2,50 \mathrm{~b}$ & $10,83 \mathrm{~b}$ & $22,49 \mathrm{bc}$ \\
Controle & $0,00 \mathrm{~b}$ & $0,00 \mathrm{~b}$ & $0,00 \mathrm{~b}$ & $0,00 \mathrm{c}$ \\
\hline
\end{tabular}

${ }^{1}$ Médias seguidas pela mesma letra, nas colunas, não diferem significativamente pelo teste de Tukey, em nível de 5\% de probabilidade.

A prospecção fitoquímica realizada com o extrato etanólico obtido do caule de $C$. linearifolius revelou a presença de ácidos fixos fortes, alcaloides, catequinas, esteroides livres, flavonois, flavonas, resinas, taninos condensados e xantonas. Não foram detectadas, nesse extrato, a presença de antocianidinas, antocianinas, bases quaternárias, cumarinas, fenois, flavononois, flavanonas, 
heterosídeos cianogênico, leucoantocianidinas, quinonas, saponinas e triterpenoides (Tabela 2).

TABELA 2: Prospecção fitoquímica do extrato etanólico da casca do caule de Croton linearifolius.

\begin{tabular}{lc}
\hline \multicolumn{1}{c}{ Metabólito Secundário } & Etanólico $^{\mathbf{1}}$ \\
\hline Ácidos fixos fortes & + \\
Alcaloides & + \\
Antocianidinas & - \\
Antocianina & - \\
Bases quaternárias & - \\
Catequinas & + \\
Cumarina & - \\
Esteroides livres & + \\
Fenois & - \\
Flavonois & + \\
Flavanonois & - \\
Flavanonas & - \\
Flavonas & + \\
Heterosídeo cianogênicos & - \\
Leucoantocianidinas & - \\
Quinonas & - \\
Resina & + \\
Saponinas & - \\
Taninos condensados & + \\
Triterpenoides & + \\
Xantona & + \\
\hline
\end{tabular}

${ }^{1}$ Resultado positivo (+), Resultado negativo (-).

Após o fracionamento do extrato etanólico, registrou-se um aumento na atividade inseticida nas frações diclorometânica e hexânica (Tabela 3 e 4). Com relação à fração diclorometânica, a partir de $16 \mathrm{~h}$ de exposição foi registrada mortalidade das larvas acima de $50 \%$. Na concentração de $13,3 \mathrm{mg} / \mathrm{mL}$ obteve-se $87,50 \%$ e $99,17 \%$ de mortalidade, em 16 e $24 \mathrm{~h}$ de observação, respectivamente. Na concentração de 6,7 $\mathrm{mg} / \mathrm{mL}$ obteve-se $69,17 \%$ e $95,00 \%$ de mortalidade, com 16 e 24 h de observação, respectivamente. Não houve diferença significativa entre as concentrações de 13,3 e $6,7 \mathrm{mg} / \mathrm{mL}$ (Tabela 3). Para a fração hexânica, somente com $24 \mathrm{~h}$ de observação constatou-se mortalidade das larvas acima de $50 \%$ e a concentração de $13,3 \mathrm{mg} / \mathrm{mL}$ foi significativamente mais ativa $(69,16 \%)$, quando comparada as demais concentrações e o grupo controle (Tabela 4).
As frações acetato de etila e hidroalcóolica não se mostraram promissoras no que diz respeito a sua eficácia sobre larvas de Aedes aegypti (Tabelas 5 e 6).

TABELA3: Percentual de mortalidade de larvas de terceiro instar de Aedes aegypti, em relação ao tempo de exposição às diferentes concentrações (mg/ $\mathrm{mL}$ ), da fração diclorometânica obtida a partir do fracionamento do extrato etanólico do caule de Croton linearifolius.

\begin{tabular}{ccccc}
\hline $\begin{array}{c}\text { Concentrações } \\
(\mathbf{m g} / \mathbf{m L})\end{array}$ & \multicolumn{4}{c}{ Mortalidade (\%) } \\
\hline & $4 \mathrm{~h}$ & $8 \mathrm{~h}$ & $16 \mathrm{~h}$ & $24 \mathrm{~h}$ \\
\cline { 2 - 5 } 13,3 & $16,67 \mathrm{a}$ & $44,17 \mathrm{a}$ & $87,50 \mathrm{a}$ & $99,17 \mathrm{a}$ \\
6,7 & $6,67 \mathrm{~b}$ & $21,66 \mathrm{~b}$ & $69,17 \mathrm{ab}$ & $95,00 \mathrm{ab}$ \\
4,0 & $1,67 \mathrm{~b}$ & $8,34 \mathrm{bc}$ & $44,17 \mathrm{bc}$ & $83,35 \mathrm{bc}$ \\
2,0 & $0,83 \mathrm{~b}$ & $3,33 \mathrm{c}$ & $22,50 \mathrm{~cd}$ & $77,50 \mathrm{c}$ \\
0,7 & $0,00 \mathrm{~b}$ & $0,83 \mathrm{c}$ & $10,00 \mathrm{~d}$ & $30,00 \mathrm{~d}$ \\
Controle & $0,00 \mathrm{~b}$ & $0,00 \mathrm{c}$ & $0,00 \mathrm{~d}$ & $0,00 \mathrm{e}$ \\
\hline
\end{tabular}

${ }^{1}$ Médias seguidas pela mesma letra, nas colunas, não diferem significativamente pelo teste de Tukey, em nível de 5\% de probabilidade.

TABELA 4: Percentual de mortalidade de larvas de terceiro instar de Aedes aegypti, em relação ao tempo de exposição às diferentes concentrações (mg/ $\mathrm{mL}$ ), da fração hexânica obtida a partir do fracionamento do extrato etanólico do caule de Croton linearifolius.

\begin{tabular}{ccccc}
\hline $\begin{array}{c}\text { Concentrações } \\
(\mathbf{m g} / \mathbf{m L})\end{array}$ & \multicolumn{4}{c}{ Mortalidade (\%) } \\
\hline & $4 \mathrm{~h}$ & $8 \mathrm{~h}$ & $16 \mathrm{~h}$ & $24 \mathrm{~h}$ \\
\cline { 2 - 5 } 13,3 & $17,50 \mathrm{a}$ & $31,67 \mathrm{a}$ & $47,50 \mathrm{a}$ & $69,16 \mathrm{a}$ \\
6,7 & $5,00 \mathrm{~b}$ & $10,00 \mathrm{~b}$ & $17,50 \mathrm{~b}$ & $27,50 \mathrm{~b}$ \\
4,0 & $3,33 \mathrm{~b}$ & $11,67 \mathrm{~b}$ & $18,33 \mathrm{~b}$ & $25,83 \mathrm{~b}$ \\
2,0 & $3,33 \mathrm{~b}$ & $4,17 \mathrm{bc}$ & $11,67 \mathrm{bc}$ & $17,50 \mathrm{~b}$ \\
0,7 & $1,67 \mathrm{~b}$ & $1,67 \mathrm{c}$ & $3,33 \mathrm{~cd}$ & $5,83 \mathrm{c}$ \\
Controle & $0,00 \mathrm{~b}$ & $0,00 \mathrm{c}$ & $0,00 \mathrm{~d}$ & $0,00 \mathrm{c}$ \\
\hline
\end{tabular}

${ }^{1}$ Médias seguidas pela mesma letra, nas colunas, não diferem significativamente pelo teste de Tukey, em nível de $5 \%$ de probabilidade. 
TABELA 5: Percentual de mortalidade de larvas de terceiro instar de Aedes aegypti, em relação ao tempo de exposição às diferentes concentrações (mg/ $\mathrm{mL}$ ), da fração acetato de etila obtida a partir do fracionamento do extrato etanólico do caule de Croton linearifolius.

\begin{tabular}{ccccc}
\hline $\begin{array}{c}\text { Concentrações } \\
(\mathbf{m g} / \mathbf{m L})\end{array}$ & \multicolumn{4}{c}{ Mortalidade (\%) ${ }^{\mathbf{1}}$} \\
\hline & $4 \mathrm{~h}$ & $8 \mathrm{~h}$ & $16 \mathrm{~h}$ & $24 \mathrm{~h}$ \\
\hline 13,3 & 0,00 & 0,00 & 0,00 & $20,84 \mathrm{a}$ \\
6,7 & 0,00 & 0,00 & 0,00 & $9,17 \mathrm{ab}$ \\
4,0 & 0,00 & 0,00 & 0,00 & $7,50 \mathrm{~b}$ \\
2,0 & 0,00 & 0,00 & 0,00 & $1,67 \mathrm{~b}$ \\
0,7 & 0,00 & 0,00 & 0,00 & $2,50 \mathrm{~b}$ \\
Controle & 0,00 & 0,00 & 0,00 & $0,00 \mathrm{~b}$ \\
\hline
\end{tabular}

${ }^{1}$ Médias seguidas pela mesma letra, nas colunas, não diferem significativamente pelo teste de Tukey, em nível de $5 \%$ de probabilidade.

TABELA 6: Percentual de mortalidade de larvas de terceiro instar de Aedes aegypti, em relação ao tempo de exposição às diferentes concentrações (mg/ $\mathrm{mL}$ ), da fração hidroalcóolica obtida a partir do fracionamento do extrato etanólico do caule de Croton linearifolius.

\begin{tabular}{ccccc}
\hline $\begin{array}{c}\text { Concentrações } \\
(\mathbf{m g} / \mathbf{m L})\end{array}$ & \multicolumn{4}{c}{ Mortalidade (\%) } \\
\hline & $4 \mathrm{~h}$ & $8 \mathrm{~h}$ & $16 \mathrm{~h}$ & $24 \mathrm{~h}$ \\
\cline { 2 - 5 } 13,3 & 0,00 & 0,00 & $3,33 \mathrm{a}$ & $5,83 \mathrm{a}$ \\
6,7 & 0,00 & 0,00 & $0,83 \mathrm{ab}$ & $5,00 \mathrm{ab}$ \\
4,0 & 0,00 & 0,00 & $0,00 \mathrm{~b}$ & $5,00 \mathrm{ab}$ \\
2,0 & 0,00 & 0,00 & $0,00 \mathrm{~b}$ & $2,50 \mathrm{ab}$ \\
0,7 & 0,00 & 0,00 & $0,00 \mathrm{~b}$ & $0,00 \mathrm{~b}$ \\
Controle & 0,00 & 0,00 & $0,00 \mathrm{~b}$ & $0,00 \mathrm{~b}$ \\
\hline
\end{tabular}

${ }^{1}$ Médias seguidas pela mesma letra, nas colunas, não diferem significativamente pelo teste de Tukey, em nível de 5\% de probabilidade.

A análise da concentração letal 50 do extrato etanólico $\left(\mathrm{CL}_{50}=17,42 \mathrm{mg} / \mathrm{mL}\right)$ e das frações hexânica $\left(\mathrm{CL}_{50}=9,58 \mathrm{mg} / \mathrm{mL}\right)$ e diclorometânica $\left(\mathrm{CL}_{50}=1,13\right.$ $\mathrm{mg} / \mathrm{mL}$ ), revelou um aumento na efetividade inseticida a medida em que o extrato etanólico foi fracionado, revelando, ainda, a fração diclorometânica como a mais ativa (Tabela 7).
TABELA 7: Concentração letal 50 do extrato etanólico do caule de Croton linearifolius e das frações hexânica e diclorometânica, sobre larvas de terceiro instar de Aedes aegypti, após $24 \mathrm{~h}$ de observação.

\begin{tabular}{lcc}
\hline \multirow{2}{*}{$\begin{array}{c}\text { Extratos } \\
\text { e frações }\end{array}$} & \multicolumn{2}{c}{ Concentração letal $(\mathbf{m g} / \mathbf{m L})$} \\
\cline { 2 - 3 } Etanólico & 17,42 & Intervalo de confiança \\
Hexânica & 9,58 & $13,69-23,89$ \\
Diclorometânica & 1,13 & $8,55-10,94$ \\
\hline
\end{tabular}

\section{Discussão}

Diversos estudos têm sido desenvolvidos com o objetivo de avaliar o potencial inseticida de espécies vegetais, na busca por novas substâncias que possam ser utilizadas no controle de vetores, e em especial no controle do Aedes aegypti (MORAIS et al., 2006; OLIVEIRA et al., 2006; RAHUMAN et al., 2008; SAKTHIVADIVEL; DANIEL, 2008; CARVALHO et al. 2011; entre outros), a exemplo do gênero Croton.

Lima et al. (2006), avaliando a atividade larvicida de hidrolatos obtidos por destilação a vapor de caules e folhas de quatro espécies pertencentes ao gênero Croton sobre o $A$. aegypti, registraram a atividade larvicida de Croton nepetaefolius e Croton zehntneri, bem como uma alteração no potencial larvicida dos hidrolatos quando comparadas as folhas e caules de Croton argyrophylloides e Croton sonderianus.

O efeito inseticida de Croton linearifolius sobre Cochliomyia macellaria foi avaliado por Silva et al. (2010), os quais demonstraram que o extrato etanólico do caule ocasionou $63,75 \%$ de mortalidade dos adultos, na concentração de $60 \mathrm{mg} / \mathrm{mL}$, não tendo sido observada atividade sobre ovos e pré-pupas dessa espécie.

A riqueza de metabólitos secundários em espécies do gênero Croton, também é destacada em vários estudos, a exemplo dos alcaloides, terpenoides (BLOCK et al., 2004; LIMA; PIRANI, 2008) e flavonoides (RANDAU et al., 2004). Segundo Salatino et al. (2007), os terpenoides são predominantes no gênero, especialmente os diterpenoides, assim como diferentes classes de alcaloides. 
A presença de alcaloides, flavonoides e taninos no extrato etanólico do caule de $C$. linearifolius corroboram com o estudo realizado por Silva et al. (2010). Com relação aos alcaloides esses autores, através da análise dos espectros de Ressonância Magnética Nuclear $\left(\mathrm{RMN}^{1} \mathrm{H}\right.$ e $\left.\mathrm{RMN}{ }^{13} \mathrm{C}\right)$, encontraram indícios de sua presença na fração diclorometânica. Esse componente poderá estar contribuindo para a melhor atividade inseticida da fração diclorometânica sobre as larvas de $A$. aegypti, quando comparada às demais frações avaliadas. Segundo Chiesa e Moyna (2004) os alcaloides esteroidais geralmente são compostos relativamente tóxicos ou muito tóxicos, estimula o interesse pelas suas propriedades biológicas, a exemplo do seu efeito fagoinibidor e tóxico em muitos insetos.

No que diz respeito aos flavonoides, diversas funções biológicas podem ser atribuídas a esses metabólitos, como, por exemplo, a proteção contra fungos, bactérias e insetos. No extrato etanólico do caule de $C$. linearifolius foi observada a presença de flavonois e flavonas, que, de acordo com Zuanasse e Montanha (2004), são flavonoides de origem biossintética muito próxima e, geralmente, classificados juntos, uma vez que os flavonois são flavonas substituídas na posição C-3 por uma hidroxila.

A presença de taninos condensados no extrato etanólico pode estar contribuindo com a atividade larvicida observada. De acordo com Santos e Mello (2004), a sua capacidade de complexação com proteínas pode ser a base para as propriedades de controle de insetos, fungos e bactérias. Estes resultados corroboram com os obtidos por Silva et al. (2010), que registraram a presença de taninos no extrato etanólico obtido do caule de Croton linearifolius, bem como na fração diclorometânica, obtida a partir do fracionamento desse extrato.

De acordo com os resultados, é possível concluir que as frações diclorometânica e hexânica, obtidas a partir do fracionamento do extrato etanólico do caule de Croton linearifolius, demonstraram-se potencialmente promissores no que diz respeito ao controle de larvas de A. aegypti.

\section{Agradecimentos}

À Fundação de Amparo à Pesquisa do Estado da Bahia e ao Banco do Nordeste do Brasil.

\section{Referências}

BLOCK, S.; BACCELLI, C.; TINANT, B.; VAN MEERVELT, L.; ROZENBERG, R.; HABIB JIWAN, J. L.; LLABRÈS, G.; DE PAUW-GILLET, M. C. Diterpenes from the leaves of Croton zambesicus. Phytochemistry, Leiden, v. 65, n. 8, p. 1165-1171, 2004.

BRASIL. Plano de manejo da Floresta Nacional Contendas do Sincorá. Informações Gerais sobre a Floresta. Vol. 1. Brasília: MMA/IBAMA, 2006. 132 p.

CARVAlHO, G. H.; SILVA, H. H. G.; CUNHA, C.; SILVA, I. G. Atividade inseticida do extrato bruto etanólico de Persea americana (Lauraceae) sobre larvas e pupas de Aedes aegypti (Diptera, Culicidae). Revista de Patologia Tropical, Goiânia, v. 40, n. 4, p. 348-361, 2011.

CHIESA, F. A. F.; MOYNA P. Alcalóides esteroidales. In: SIMÕES, C. M. O.; SCHENKEL, E. P.; GOSMANN, G.; MELLO, J. C. P., MENTZ, L. A.; PETROVICK, P. R. (Ed.). Farmacognosia: da planta ao medicamento. 5 ed. Porto Alegre: Editora da UFRGS, 2004. p. 869-883.

HORTA, M. A. P.; CASTRO, F. I.; ROSA, C. S.; DANIEL, M. C.; MELO, A. L. Resistance of Aedes aegypti (L.) (Diptera: Culicidae) to Temephos in Brazil: A Revision and New Data for Minas Gerais State. BioAssay, Piracicaba, v. 6, n. 7, p. 1-6, 2011.

LIMA, L. R.; PIRANI J. R. Revisão taxonômica de Croton sect. Lamprocroton (Mull.Arg.) Pax (Euphorbiaceae s.s). Biota Neotropica, Campinas, v. 8, n. 2, p. 177-230, 2008.

LIMA, M. G.; MAIA, I. C.; SOUSA, B. D.; MORAIS, S. M.; FREITAS, S. M. Effect of stalk and leaf extracts from Euphorbiaceae species on Aedes aegypti (Diptera, Culicidae) larvae. Revista do Instituto de Medicina Tropical de São Paulo, São Paulo, v. 48, n. 4, p. 211-214, 2006.

MATOS, F. J. A. Introdução à fitoquímica experimental. 2 ed. Fortaleza: Editora da UFC. 1988. 141 p.

MORAIS, S. M.; CAVALCANTI, E. S. B.; BERTINI, L. M.; OLIVEIRA, C. L. L.; RODRIGUES, J. R. B.; LEAL-CARDOSO, J. H. Larvicidal activity of essential oils from brazilian Croton species against Aedes aegypti L. Journal of the American Mosquito Control Association, Washington, v. 22, n. 1, p. 161-164, 2006.

OLIVEIRA, A. M.; HUMBERTO, M. M. S.; SILVA, J. M.; ROCHA, R. F. A.; SANT'ANA. Estudo fitoquímico e avaliação das atividades moluscicida e larvicida dos extratos da casca do caule e folha de Eugenia malaccensis L. (Myrtaceae). Revista Brasileira de Farmacognosia, Curitiba, v. 16, p. 618-624, 2006.

OPS/OMS. Dengue: guías para el diagnostico, tratamento, prevención y control. La Paz: OPS/OMS, 2010. 152 p.

PROPHIRO, J. S.; SANTOS SILVA, O.; LUNA, J. E. D.; PICCOLI, C. F.; KANIS, L. A.; NAVARRO DA SILVA, N. A. Aedes aegypti and Aedes albopictus (Diptera: Culicidae): coexistence and susceptibility to temephos, in municipalities with occurrence of 
dengue and differentiated characteristics of urbanization. Revista da Sociedade Brasileira de Medicina Tropical, Uberaba, v. 44, n. 3, p. 300-305, 2011.

RAHUMAN, A.A.; GOPALAKRISHNAN, G.; VENKATESAN, P.; GEETHA, K. Larvicidal activity of some Euphorbiaceae plant extracts against Aedes aegypti and Culex quinquefasciatus (Diptera: Culicidae). Parasitology Research, Heidelberg, v. 102, n. 5, p. 867-873, 2008.

RANDAU, K. P.; FLORÊNCIO, D. C.; FERREIRA, C. P.; XAVIER, H. S. Estudo farmacognóstico de Croton rhamnifolius H.B.K. e Croton rhamnifolioides Pax \& Hoffm. (Euphorbiaceae). Revista Brasileira de Farmacognosia, Curitiba, v. 4, n. 2, p. 8996, 2004.

SAKTHIVADIVEL, M.; DANIEL, T. Evaluation of certain insecticidal plants for the control of vector mosquitoes viz. Culex quinquefasciatus, Anopheles stephensi and Aedes aegypti. Applied Entomology and Zoology, Tokyo, v. 43, n. 1, p. 57-63, 2008.

SALATINO, A.; SALATINO, M. L. F.; NEGRI, G. Traditional uses, Chemistry and pharmacology of Croton species (Euphorbiaceae). Journal of the Brazilian Chemical Society, São Paulo, v. 18, n. 1, p. 11-33, 2007.

SANTOS, S. C.; MELlO, J. C. P. Taninos. In: In: SIMÕES, C. M. O.; SCHENKEL, E. P.; GOSMANN, G.; MELLO, J. C. P., MENTZ, L. A.; PETROVICK, P. R. (Ed.). Farmacognosia: da planta ao medicamento. 5 ed. Porto Alegre: Editora da UFRGS, 2004. p. 615-656.
SILVA, S. L. C.; CARVALHO, M. G.; GUALBERTO, S. A.; CARNEIRO-TORRES, D. S.; VASCONCELOS, K. C.; OLIVEIRA, N. F. Bioatividade do extrato etanólico do caule de Croton linearifolius Mull. Arg. (Euphorbiaceae) sobre Cochliomyia macellaria (Diptera: Calliphoridae). Acta Veterinaria Brasilica, Feira de Santana, v. 4, n. 4, p. 252-258, 2010.

WHO. Dengue and dengue haemorrahagic fever. Geneva: World Health Organization, 2008. Factsheet no 117.

WHO. Temephos in drinking-water: use for vector control in drinking-water sources and containers. Geneva: World Health Organization, 2009. Background document for development of WHO Guidelines for Drinking-water Quality.

WHO. Global strategy for dengue prevention and control 20122020. Geneva: World Health Organization, 2012.

ZUANAZZI, J. A. S.; MONTANHA, J. A. Flavonóides. In: In: SIMÕES, C. M. O.; SCHENKEL, E. P.; GOSMANN, G.; MELLO, J. C. P., MENTZ, L. A.; PETROVICK, P. R. (Ed.). Farmacognosia: da planta ao medicamento. 5. ed. Porto Alegre: Editora da UFRGS, 2004. p. 577-614. 